\title{
La force de la désobéissance : retour sur la chute du régime de Ben Ali
}

\author{
Larbi Chouikha* et Éric Gobe*
}

Au milieu des années 2000, la lecture de certains travaux de science politique donnait à penser que le régime mis en place par le président Ben Ali paraissait devoir durer. Béatrice Hibou (2006) évoquait la «force de ľobéissance» et la règle de "laccommodement négocié » qui, telles une loi d airain, régissaient les relations entre la population tunisienne et le pouvoir politique. Toutefois, bien que faisant référence aux travaux de Michel Foucault, lauteur ignorait les formes de «contre-conduites» ou de «résistances» définissant le passage au politique de certains secteurs ou acteurs de la société tunisienne (Camau, 2008).

Or depuis le milieu des années 2000, la question lancinante de la succession dun président Ben Ali malade et les ambitions de la belle famille Trabelsi aspirant d une façon ou $\mathrm{d}$ une autre à prendre sa place posaient la question de la durabilité du régime. La perspective de voir la coterie mafieuse entourant le président Ben Ali accéder directement au sommet de 1 État apparaissait insupportable aux différentes catégories sociales de la population tunisienne, y compris chez une partie de ľoligarchie au pouvoir pour qui les Trabelsi n étaient que des parvenus. Dans une telle configuration, la base de légitimité du régime paraissait de plus en plus friable.

Par ailleurs, l冖affaiblissement de la capacité redistributive de 1̌État tunisien consécutive à la crise financière internationale de 2008 et la dégradation de la situation sociale, notamment celle des diplômés originaires de lintérieur fortement touchés par le chômage, conduisaient certains chercheurs à s interroger sur les contours d un mouvement social qui serait susceptible d ébranler les bases du régime et d inaugurer une « ouverture de la structure des opportunités politiques » (Chouikha et Gobe, 2009). Certes, les mouvements protestataires apparaissaient comme sectoriels, les politiques de dépolitisation et de coercition menée par le pouvoir de Ben Ali empêchant le développement de «mobilisations multisectorielles » (Dobry, 1986), qui auraient pu déstabiliser le régime, comme cela avait été le cas à la fin du règne de Bourguiba.

Toutefois, la révolte du bassin minier de Gafsa était révélatrice d une mobilisation sociale inédite dans la mesure où le régime de Ben Ali avait mis plus de six mois à la réduire. Ce mouvement de Gafsa donnait «à penser que le `pays de 1 ‘intérieur_» pourrait «jouer aussi un rôle dans la redéfinition des enjeux politiques futurs et la transition vers un nouveau type de régime ». En fait, la machine sécuritaire tunisienne confrontée à une « révolte par le bas », animée par des citoyens ordinaires, a révélé les difficultés du régime autoritaire tunisien à faire face à un mouvement de protestation populaire. Cette révolte était «le signe dun ébranlement du Pacte de sécurité, ce contrat social implicite entre 1ヒ́tat et le peuple, que certains auteurs considéraient comme le principal moteur du régime de Ben Ali » (Chouikha et Geisser, 2010).

Par certaines de ces dimensions le mouvement protestataire du la région de Gafsa apparaît comme une anticipation du soulèvement populaire qui a conduit au départ du président Ben Ali. Une grande partie de la population avait le sentiment que le bassin minier $\mathrm{n}$ avait pas bénéficié de la même sollicitude de la part du pouvoir central que d autres régions, notamment le Sahel, principal pourvoyeur en élites politiques et économiques tunisiennes. Les « laissés pour compte du marché du travail », i.e. les diplômés chômeurs, les travailleurs précaires et les veuves de mineurs se sont mobilisés pour réclamer la justice sociale et la dignité (al-karama). Formuler de telles revendications revenait à dénoncer le népotisme, ainsi

\footnotetext{
${ }^{*}$ Professeur à 1 Tnstitut de presse et des sciences de 1 innformation (IPSI), Université de la Manouba.

* Chercheur à 1 TREMAM/CNRS, rédacteur en chef et responsable scientifique de L $\breve{A n n e ́ e ~ d u ~ M a g h r e b . ~}$
} 
que les pratiques clientélistes et tribales des dirigeants locaux du RCD et de 1UGTT dans la distribution des emplois. Toutefois, la révolte du bassin minier n a pas débouché sur un changement de régime. Peu relayée par les médias occidentaux et tunisiens, la mobilisation est restée cantonnée à la région de Gafsa et a connu relativement peu décho sur internet, bien que certains sites 1 aaient médiatisée. Face à la répression et en 1 absence de soutiens suffisamment solides dans la société, lépisode conflictuel de Gafsa est retombé et n a pu déboucher ni sur un mouvement social durable, ni a fortiori sur le renversement du régime, à la différence de lépisode protestataire initiée à Sidi Bouzid le 17 décembre 2010.

\section{Une révolte sociale spontanée ? Le rôle joué par I Union générale tunisienne du travail dans la mobilisation}

Le premier adjectif utilisé par les journalistes pour qualifier le mouvement protestataire né à Sidi Bouzid est "spontané ». L élément déclencheur des mobilisations qui ont mené à la chute de régime de Ben Ali est tout à fait identifiable. C est 1 ‘immolation par le feu de Mohamed Bouazizi, vendeur de fruits et légumes à la sauvette, à Sidi Bouzid, agglomération de 40000 habitants située au centre de la Tunisie, région parmi les plus pauvres de Tunisie.

Les maîtres mots qui permettent de comprendre la déclanchement de la révolte sont 1 humiliation et 1 ¿̌ndignation. Humilié Mohamed Bouazizi l`est : non seulement, la police lui a confisqué son outil de travail (sa charrette et sa marchandise) mais de surcroît une employée municipale lă giflé, le renvoyant ainsi à sa condition indigne. Dès le 17 décembre, des commerçants et des dizaines de jeunes indignés se réunissent devant le siège du gouvernorat de Sidi Bouzid pour organiser un sit-in de protestation. Durant le week-end du 18 et du 19 décembre, les rassemblements de jeunes se multiplient, tandis que les accrochages avec les forces de lordre qui tentent de disperser la foule s intensifient et que les premières arrestations sont effectuées. Le suicide d un autre jeune, diplômé chômeur, par électrocution le 22 décembre, relance la révolte sociale qui s étend aux petites villes voisines de Sidi Bouzid, Meknassy et surtout de Menzel Bouzaïane, agglomération dans laquelle les émeutiers incendient le siège de la délégation et assiègent le poste de la garde nationale (l équivalent de la gendarmerie). Le 24 décembre 1 ¿insurrection sociale se propage dans le centre du pays. Le jour même, Mohamed Laamari, un jeune diplômé chômeur est tué d une balle dans la poitrine à Menzel Bouzaïane. Les violences policières et les arrestations massives suscitent de nouvelles manifestations et émeutes qui débouchent sur de nouveaux heurts avec les forces de sécurité. Les slogans alors scandés par les manifestants ont une forte connotation sociale, même si parfois 1 on voit poindre un arrière plan politique ${ }^{1}$. À partir de la fin décembre, les mots d ordre scandés par les manifestants prennent une connotation éminemment politique et c est désormais la personne du président Ben Ali qui est visée $^{2}$.

Si dans ce premier temps de la révolte, la direction de 1 Union générale tunisienne du travail (UGTT), largement inféodée au pouvoir politique, demeure attentiste, certains militants de base et cadres des Unions régionales et locales accompagnent le mouvement en organisant des manifestations de solidarité avec les émeutiers.

\footnotetext{
${ }^{1}$ « L ěmploi est un droitǔ Bande de voleurs », «Citoyens venez avec nousǔ Le silence est la pire des trahisons », « honte au gouvernementǔ les prix flambent», «Emploi, liberté, dignité », «à bas le Rassemblement [les parti politique présidé par Ben Ali], « Non aux Trabelsi, pilleurs des deniers publics », http://www.assabilonline.net, 24 décembre 2010, consulté le 25 janvier 2011 (en arabe).

2 «Pas de succession, ni de prorogation, nous sommes tous des Sidi Bouzid»; «Libertés, libertés, pas de présidence à vie ». Cf. http://jeanmarcelbouguereau.blogs.nouvelobs.com/archive/2010/12/29/ tunisie-la-revolteest-en-train-de-changer-de-nature.html, consulté le 5 janvier 2011.
} 
La centrale syndicale indépendamment de lăllégeance affichée par sa direction à légard du chef de 1 État est la seule organisation avec le parti de Ben Ali (le Rassemblement constitutionnel démocratique ${ }^{-} \mathrm{RCD}$ ) à disposer dune implantation nationale. Des militants syndicaux organisent à partir des locaux des Unions régionales de 1UGTT des sit-in et des marches pour dénoncer la répression policière, la corruption, le développement inégal entre les régions, la marginalisation des jeunes et 1 appauvrissement de la masse de la population ${ }^{3}$. Certains syndicats de branche qui avaient su préserver une partie de leur autonomie sous la présidence de Ben Ali viennent renforcer l entrée progressive de 1 UGTT dans le mouvement protestataire : à lăappel des syndicats de la sécurité sociale, de la santé, de la poste, de lenseignement secondaire des centaines de manifestants se rassemblent dans la capitale, Tunis, sur la place Mohamed Ali devant le siège de 1 UGTT $^{4}$.

$\mathrm{C}$ `est à la suite de la répression brutale des manifestations de Kasserine (chef lieu du gouvernorat voisin de Sidi Bouzid, plus à l est) des 7, 8 et 9 janvier qui font officiellement 14 morts (environ 50 officieusement) que la direction de la centrale syndicale prend conscience de la dynamique de laction collective qui a pris un tournant radical : 1 insurrection s est propagée à l lensemble des centres urbains du pays, capitale comprise et les «classes moyennes " - autrement dit les membres de professions intellectuelles comme les avocats, les enseignants, les médecins, etc. - alimentent les manifestations constituées à ľorigine de jeunes des quartiers populaires, souvent diplômés chômeurs.

Les slogans portés par les protestataires appellent alors explicitement au " départ de Ben Ali et de sa clique de voleurs ». Aussi, la commission administrative de 1UGTT, sous la pression des Union régionales et locales, décide-t-elle le 11 janvier d autoriser les Unions régionales à organiser des grèves générales sur lensemble du territoire tunisien le lendemain pour « protester contre les tirs à balles réels sur les citoyens de Sidi Bouzid et Kasserine ». Quant à Tunis, la grève générale est prévue pour le 14 janvier $^{5}$. Alors que le 11 janvier, des émeutes éclatent dans les quartiers populaires d Ettadhamen et Mnihla, la grève générale du 12 janvier est un succès dans toutes les agglomérations, notamment a à Sfax, la deuxième ville du pays. Ce faisant 1 UGTT donne un tournant décisif au mouvement protestataire qui débouche sur la journée du 14 janvier qui voit Ben Ali quitter le pouvoir : dans le sillage de la grève générale, un défilé comptant des centaines de milliers de personnes de toutes catégories sociales défilent sur lavenue Habib Bourguiba entre la porte de France et le ministère de 1 Intérieur au cri de «Ben Ali dégage ».

Mais la Centrale syndicale nă pas été lăacteur du "changement d échelle » de lépisode conflictuel né à Sidi Bouzid. Elle a accompagné et parachevé l`action collective plus qu elle ne lui a permis de prendre une envergure nationale. Le mouvement protestataire tunisien a en fait emprunté « la voie de la diffusion directe », celle " opérée par des individus ou des groupes dont les similitudes ou les relations préexistantes deviennent la base de la mobilisation » (Tilly et Tarrow, 2008, p. 164). En fait, le mouvement protestataire tunisien qui a conduit à la chute du président Ben Ali se caractérise par l ỉnexistence de leaders. Cette absence d entrepreneurs de mobilisation $\mathrm{n} \breve{a}$ pas empêché le mouvement de protestation de se politiser et de changer d échelle. L usage des nouvelles technologies de l information a probablement pallié cette absence de leadership en servant de caisse de résonnance au mouvement protestataire.

\footnotetext{
${ }^{3}$ Le 25 décembre voit l`entrée en jeu des militants de base de 1 UGTT dans de nombreuses agglomérations, non seulement dans le gouvernorat de Sidi Bouzid (Tala, Regueb, Meknassy, Souk el Jedid et Bir el Hfay), mais aussi à Kasserine, Gafsa et Tunis. Cf. Al badil Ajil, 25 décembre 2010, sur le site du Parti communiste des ouvriers de Tunisie. http://www.albadil.org/, consulté le 1er février 2011 (en arabe).

${ }^{4}$ AP, 27 décembre 2010.

${ }^{5} \mathrm{http}: / /$ www.assabilonline.net, 11 janvier 2011, consulté le $1^{\mathrm{er}}$ février 2011 (en arabe).
} 


\section{La Tunisie virtuelle : caisse de résonance du mouvement protestataire}

L usage d internet comme instrument de protestation contre le régime de Ben Ali ne date du 17 décembre 2010. À côté dǔun espace public, complètement domestiqué par le pouvoir politique où les possibilités d expression étaient quasiment nulles, šest développée, avec le web, une Tunisie virtuelle dans laquelle les internautes tunisiens utilisaient des proxys qui leur permettaient de contourner la censure gouvernementale d internet. Grâce à ces outils, les Tunisiens de toutes catégories sociales pouvaient, plus ou moins, naviguer sur les sites interdits, échangeaient des informations de toute sorte et diffusaient des vidéos sur les sites de partage comme YouTube ou Dailymotion ${ }^{6}$. Mais avec le développement des réseaux sociaux et laccroissement du nombre d internautes, le web est devenu un espace autonome $\mathrm{d}$ expression et de socialisation politique d une partie de la jeunesse tunisienne ${ }^{7}$.

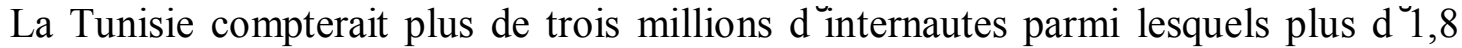
millions disposeraient $d$ un compte Facebook. Or le développement du célèbre réseau social en Tunisie depuis trois ans est luun des faits les plus notables de lévolution de lusage $\mathrm{d}$ internet dans le pays. Cette nouvelle « arène médiatique » a contribué à la « mobilisation de laction» (Granjon, 2009, p. 355). Dès le début du mouvement protestataire, les jeunes internautes de Sidi Bouzid ont diffusé de nombreux documents audiovisuels produits de 1 usage des téléphones portables munis de caméra et d appareils photo sur le réseau social Facebook et sur le site Youtube. Les journalistes ne pouvant se rendre sur place en raison du blocage de la région par les forces de police, les médias satellitaires ${ }^{8}$ ont abondamment utilisé dans leurs reportages la matière première diffusée sur Facebook ou le site YouTube.

Les internautes de Sidi Bouzid, puis du reste de la Tunisie, au fur et à mesure que le mouvement protestataire prenait de lampleur, sont devenus des producteurs et diffuseurs d informations. Ils ont ainsi créé des comptes sur le réseau social Facebook consacrés spécifiquement à la couverture quasiment instantanée des évènements protestataires. Ce faisant, Facebook et les sites de cyberdissidents tunisiens ont ouvert la voie un nouveau processus collectif d énonciation des enjeux du mouvement protestataire. Par le truchement du célèbre réseau social, les internautes ont non seulement produit et diffusé des informations, mais ont également lancé des appels à manifestations, des mots d ordre de grève qui ont contribué à faire changer $\mathrm{d}$ échelle au mouvement protestataire. Ils ont relayé des messages de plus en plus radicaux appelant à rompre avec le régime de Ben Ali. Autrement dit, la force d agrégation du réseau social a donné naissance à une forme inédite de mobilisation, certains internautes devenant des entrepreneurs de mobilisation dont lactivité virtuelle sur le web $\mathrm{s}$ est traduite dans la réalité par diverses formes d action collective (manifestations, grèves, sit-in, etc.). Le pouvoir politique a pris conscience de la capacité de certains internautes à

\footnotetext{
${ }^{6}$ Les pouvoirs publics tentaient, tant bien que mal de limiter cet espace d autonomie par la censure. Le censeur ${ }^{-}$ les services spécialisés du ministère de 1 Intérieur - était surnommé, sur le mode humoristique « Ammar 404 » par référence aux erreurs http 404 qui s affichaient lorsque les internautes présents sur le territoire tunisien souhaitaient accéder à des ressources web interdites par le gouvernement tunisien. Par ailleurs, les blogueurs, les journalistes et autres militants des droits de 1 Homme étaient souvent victimes de vol d identifiants accompagné du piratage de leur messagerie électronique. «Le blog `débat Tunisie_ Nouvelle victime de la censure de l“ATI » sur http://www.attariq.org/spip.php?article273, consulté le 10 février 2011.

${ }^{7}$ Parmi les sites pionniers, on citera Takriz (ras-le-bol en dialecte tunisien) lancé par de jeunes internautes vivant pour la plupart sur le territoire tunisien et exprimant un profond malaise dans la jeunesse. On peut également évoquer Tunezine créé en 2000 par Zouhayr Yahyaoui, premier internaute à avoir été incarcéré et condamné à deux ans de prison ferme pour "propagation de fausses nouvelles » et "utilisation frauduleuse délibérée de lignes de communication ». Par ailleurs, la publication sur internet de la lettre de diffusion critique du pouvoir tunisien, Tunisnews, est 1 une des illustrations les plus probantes de 1 ćmergence d un espace d autonomie, d expression et de diffusion de 1 information politique sur le régime Ben Ali et ses turpitudes (Chouikha, 2009).

${ }^{8}$ Notamment Al Jazeera. Cf. «Sidi Bouzid sur Facebook », 25 décembre 2010, http://www.aljazeera.net/, consulté le 10 janvier 2011 (en arabe).
} 
lancer des mots d ordre et à être relayés sur le terrain par les membres tunisiens du réseau social. Aussi a-t-il procédé à lăarrestation de jeunes blogueurs et cyberdissidents particulièrement actifs sur Facebook tout en censurant les sites d opposition ${ }^{9}$.

Pour les tunisiens n ayant pas accès au "réseau des réseaux », Al Jazeera a servi de substitut aux sites internet en diffusant quasiment en continu depuis le 24 décembre des images du mouvement protestataire. De manière générale, les images d immolation par le feu, la vision des morts et des blessés par balles ont très probablement contribué, par 1 indignation qu elles ont suscitée, à faire tomber la peur. L indice le plus probant de ce lien de causalité est donné par le changement d échelle du conflit qui a suivi la diffusion des images extrêmement violentes de la répression de Kasserine (notamment les scènes filmées dans les hôpitaux, à la limite du supportable où 1 on voit des cadavres éventrés et des cranes éclatés) par les brigades lordre public (BOP).

Mais l usage à grande échelle de la répression, tout comme la mise en `uvre d une politique de communication gouvernementale avec un temps de retard, a été incapable de juguler le mouvement protestataire.

\section{De la fuite en avant sécuritaire du régime à la révolution de Palais}

En dépit du quadrillage sécuritaire et du système de surveillance sophistiqué mis en place par le régime de Ben Ali, le soulèvement populaire a pris au dépourvu aussi bien les autorités que les partis reconnus de lopposition qui, il est vrai, ne sont guère représentatifs et dont les structures sont d une faiblesse extrême. Les deux piliers du système sécuritaire, le parti-État, le Rassemblement constitutionnel démocratique et les forces de police ont été débordées par l lampleur du mouvement.

Dix jours après le début du soulèvement, la politique de répression et le blackout imposé aux médias tunisiens nont pas permis le retour à lordre. Dans la mesure où la répression $\mathrm{n} \breve{a}$ pas donné les effets escomptés, le chef de 1 État a commencé à développer une politique de communication qui va se trouver sans cesse en décalage par rapport aux revendications formulées par les manifestants et les émeutiers. Le 28 décembre 2010, pour montrer toute sa compassion, il se rend à son chevet de Mohamed Bouazizi soigné au centre médical des grands brûlés à Ben Arous près de Tunis. Le même jour, dans un discours diffusé par la chaîne nationale tunisienne, le président tunisien s en prend à "une minorité d extrémistes et agitateurs » à l`encontre desquels « la loi sera appliquée en toute fermeté ». Il dénonce les chaînes de télévision étrangères qu il accuse de diffuser des "allégations mensongères sans vérification ». Son discours n empêche pas les émeutes et les manifestations de gagner d autres villes de province. La décision de remanier le gouvernement le lendemain, ainsi que la mutation des gouverneurs de Sidi Bouzid, Jendouba et Zaghouan décidée le 30 décembre, apparaissent dérisoires au regard des revendications de plus en plus politiques portées par les manifestants. Au début du mois de janvier, la proposition gouvernementale de créer de grands projets d investissement dans le gouvernorat de Sidi Bouzid ne jugule en rien la dynamique des protestations dont le centre de gravité de déplace vers Kasserine ${ }^{10}$. La ville connaît une véritable atmosphère de guérilla urbaine alourdie par les pillages de magasins, les tirs de snipers et les descentes de police dans les domiciles des familles des supposés émeutiers. La deuxième intervention télévisée du chef de

\footnotetext{
9 Boris Manenti, «Des blogueurs arrêtés après les troubles de Sidi Bouzid», http://tempsreel.nouvelobs.com/actualite/monde/20110107.OBS5863/des-blogueurs-tunisiens-arretes-apres-lestroubles-de-sidi-bouzid.html, consulté le 31 janvier 2011.

${ }^{10}$ Michael Ayari et Vincent Geisser, «Tunisie : la Révolution des 'Nouzouh_ n a pas 1 ${ }^{\circ}$ odeur du jasmin », Témoignage chrétien, 25 janvier 2011.
} 
1 État le 10 janvier qui dénonce des «voyous cagoulés aux actes terroristes impardonnables [ǔ ] à la solde de l'étranger, qui ont vendu leur âme à l'extrémisme et au terrorisme » et annonce la création de trois cent mille emplois en deux ans ne fait pas retomber la tension. La décision de fermer temporairement les établissements scolaires et les universités montre un régime aux abois qui veut éviter que les campus et les lycées soient des lieux d amplification de la contestation ${ }^{11}$. Alors que les émeutes ont atteint le $\mathrm{c}^{`}$ ur de la capitale, le limogeage le 12 janvier du ministre de 1 Intérieur Rafik Belhaj Kacem, ainsi que la libération des personnes arrêtées depuis le début de lépisode protestataire, ne contribue en rien à stopper le mouvement insurrectionnel qui est désormais appuyé par la direction de 1 UGTT.

Le discours télévisé du 13 janvier donne à voir un chef de 1 État sous pression qui semble céder à toutes les revendications des Tunisiens descendus dans la rue, à l exception de son départ du pouvoir. Depuis le matin, les petits partis de lopposition légale critiques (le Parti démocrate progressiste, le Tajdid et le Forum démocratique pour le travail et les libertés) négocient avec le Premier ministre Mohamed Ghannouchi la constitution d un gouvernement d unité nationale. De plus, le soir de la diffusion du discours télévisé, la chaîne nationale tunisienne organise un débat avec, pour la première fois dans la Tunisie de Ben Ali, des représentants $\mathrm{d}$ organisations non gouvernementales d opposition - notamment en présence de Mokhtar Trifi, le président de la Ligue tunisienne de défense des droits de 1 Homme.

Mais ce discours et les négociations avec les partis de lopposition critique arrivent trop tard : la pression populaire est trop forte et une partie des élites au pouvoir n est plus prête à cautionner la politique répressive du pouvoir. En effet, la force du mouvement de protestation ne doit pas nous faire perdre de vue que la « révolution populaire » s articule à une « révolution de palais ». Le départ du président Ben Ali est également le résultat d" "une fracture dans loligarchie au pouvoir $»^{12}$.

Dans cette configuration, l冖armée et son chef d état major ont joué un rôle décisif dans le départ du président Ben Ali. Cette intervention des militaires ressortit apparemment à un paradoxe. L ăarmée tunisienne, contrairement à 1־Algérie ou 1 ̌́gypte, na jamais constitué lépine dorsale du régime politique tunisien (Camau et Geisser, 2003, p. 207-211). Armée de conscription, marginalisée sous les présidences de Bourguiba et de Ben Ali, elle dispose dun effectif faible (35000 hommes à comparer aux 120000 hommes des forces de sécurité) ${ }^{13}$. Si pendant la période de la présidence Ben Ali, laarmée a été modernisée et la carrière militaire revalorisée, la Tunisie $\mathrm{n} \breve{a}$ pas connu lěmergence d un appareil militaro-économique susceptible den faire un acteur économique impliqué dans laffairisme de ľentourage présidentiel. Acteur dépolitisé, elle est restée fidèle au président Ben Ali jusqu au moment où celui-ci a donné 1 ordre de tirer sur les manifestants. Elle a alors refusé de sortir de son rôle de défense extérieure pour endosser celui de force de maintien de 1̌ordre. Le chef d état major Rachid Ammar, qui a accepté de déployer lăarmée dans les centres urbains pour calmer la situation, est démis de ses fonctions par le président Ben Ali le 11 janvier, en raison justement de son refus d ordonner de tirer sur la foule. Dans le même temps, des militaires sont vus $\mathrm{s}$ interposant entre les forces de police et les manifestants. Aussi 1 attitude de 1 ־armée se situet-elle aux antipodes de celle des forces de police restées fidèle jusqu au bout au chef de 1 État.

Bien qu assigné à résidence, le général Ammar a, semble-t-il, conseillé au président Ben Ali de partir et lui aurait fait savoir le 14 janvier qu il bénéficiait d un créneau de 3

\footnotetext{
${ }^{11}$ Il s a agit également d éviter que des enfants ou des adolescents ne tombent sous les balles des policiers avec tous les effets désastreux en termes d image pour le régime qu auraient de tels évènements.

12 Pierre-Robert Baduel, «Tunisie: le rôle complexe et déterminant de lăarmée », Le Monde.fr, http://www.lemonde.fr, 10 février 2011, consulté de le 11 février 2011.

13 Sammy Ghorbal, "Rachid Ammar, 1homme fort de la Tunisie: 'L armée ne tire pas_», http://www.rue89.com/2011/01/16/larmee-ne-tire-pas-lhomme-fort-de-la-tunisie-est-general-185923, consulté le 10 février 2011.
} 
heures pour quitter le pays avant la fermeture de lespace aérien tunisien. Toutefois, 1 institution militaire a refusé de conduire un coup d État pour prendre le pouvoir. Elle jouit depuis lors, auprès de la population, $\mathrm{d}$ un prestige $\mathrm{d}$ autant plus fort qu après le départ $\mathrm{du}$ président Ben Ali, elle s est chargé de pourchasser les milices présidentielles. Mais son rôle exact reste à apprécier. Certains éléments de 1 élite au pouvoir sous Ben Ali, - incarnés notamment par des individus originaires du Sahel comme Kamel Morjane et par le chef d état major lui-même ${ }^{-}$auraient poussé au départ du président Ben Ali pour se débarrasser de la belle famille et des membres de loligarchie les plus compromis dans le fonctionnement du régime de Ben Ali. L armée s est ainsi placée «en position de recours si un processus crédible de stabilisation politique $\mathrm{n} \breve{\text { aboutissait }}$ pas $\gg{ }^{14}$.

Cette « révolution tunisienne » a mis à mal le discours selon lequel les Tunisiens étaient avant tout des consommateurs individualistes, rentrés depuis les années 1990 dans la société de consommation peu concernés la chose politique. L épisode protestataire a suscité des manifestations de solidarité, notamment au moment de la chute du régime : les habitants des divers quartiers ont constitué des comités d autodéfense civile pour protéger les biens privés face aux attaques des pilleurs et des miliciens du parti gouvernemental. Des formes d auto-organisation ont surgi pour pallier le non fonctionnement des services publics municipaux (nettoyage des lieux publics, ramassage des orduresǔ ).

Mais lăabsence de leadership au sein du mouvement protestataire alimente l linstabilité de la Tunisie post-14 janvier. Elle contribue à créer un hiatus entre un gouvernement «transitoire » en mal de légitimité et souvent, coupé des réalités et des Tunisiens désormais prompts à descendre dans la rue pour tenter d imposer aux autorités la politique à suivre. Mis sous pression, le gouvernement de Mohamed Ghannouchi a dû, entre autres, se séparer de certains ministres trop liés à lancien régime, démettre ľensemble des gouverneurs, et suspendre les activités du RCD. Lui-même a dû démissionner le 27 février 2011. Une ligne de partage se dessine entre les soutiens du gouvernement de transition et le Conseil national de sauvegarde de la révolution créée le 11 février 2011. Les premiers sont constitués pour partie des composantes les plus technocratiques et les moins compromises de l'ancien régime, ainsi que de certains leaders de l'opposition reconnue de l'époque de Ben Ali. Ces derniers, qui s'inscrivent dans une logique de compromis, veulent des élections législatives et présidentielles anticipées, organisées dans le cadre d'une Constitution et d'un code électoral amendés.

Le second, quant à lui, est une coalition hétérogène qui rassemble 28 partis politiques, associations et organisations professionnelles. On trouve en son sein 1UGTT, le Conseil de 1 ordre des avocats, les islamistes d Ennahda, divers groupuscules d extrême gauche et des associations de défense des droits de 1 Homme et de lutte contre la torture. Ce courant, radical dans sa revendication démocratique, exige une forte épuration de l'appareil d'État et demande l'élection d'une assemblée constituante qui devra faire table rase du passé institutionnel de la Tunisie et modifier le système de fond en comble, afin de proposer une nouvelle Constitution instituant un régime parlementaire.

Cette phase transitionnelle engendre de nouvelles formes d action et stimule le débat public. Mais dans le même temps, elle recèle des risques de désordre qui peuvent susciter des contrecoups et des reprises en main de la part des certains acteurs disposant de moyens de coercition. Il serait toutefois hasardeux de se livrer plus avant à des conjectures tant la situation est exceptionnelle : tout comme la plupart des observateurs, 1 Łimmense majorité des

\footnotetext{
${ }^{14}$ Pierre-Robert Baduel, op .cit.
} 
Tunisiens ne s imaginait pas possible, avant le 17 décembre 2011, que le régime politique mis en place par Ben Ali le 7 novembre 1987 ne s effondre.

\section{Bibliographie}

CAMAU Michel, 2008, « Tunisie : vingt après. De quoi Ben Ali est-il le nom ? », L ‘nnée du Maghreb 2008, Paris, CNRS Éditions, p. 507-527.

CAmau Michel et GeISSER Vincent, 2003, Le syndrome autoritaire. Politique en Tunisie de Bourguiba à Ben Ali, Paris, Presses de Sciences Po.

CHOUIKHA Larbi, 2009, «Un cyberspace autonome dans un espace autoritaire : l'expérience de Tunisnews ", in MoHSEN-FinAN Khadija (dir.), Les médias en Méditerranée. Nouveaux médias, monde arabe et relations internationales, Arles/Aix-en-Provence, Actes Sud/MMSH, p. 217-235.

CHOUIKHA Larbi et GeISSER Vincent, 2010, « Retour sur la révolte du bassin minier. Les cinq leçons politiques dư conflit social inédit »L L Année du Maghreb, VI, 2010. Paris, CNRS Éditions, p. 415-426.

Chouikha Larbi et GoBe Éric, 2009, « La Tunisie entre la révolte du bassin minier de Gafsa et 1 échéance électorale de $2009 »$, L“Année du Maghreb 2009, Paris, CNRS Éditions, p. 387420 .

DoBry Michel, 1986, Sociologie des crises politiques, Paris, Presses de Sciences Po.

Granjon Fabien, 2009, « Média », in Fillieule Olivier, Mathieu Lilian et Pechu Cécile, Dictionnaire des mouvements sociaux, Paris, Les Presses de Sciences po, p. 349-355.

HiBou Béatrice, 2006, La force de ľobéissance. Luéconomie de la répression en Tunisie, Paris, La Découverte.

TARrow Sidney et Tilly Charles, 2008, Politique(s) du conflit. De la grève à la révolution, Paris, Les Presses de Sciences po. 